ARTICLE

DOI: $10.1038 / \mathrm{s} 41467-018-04059-1$

\title{
Evolution of high-level resistance during low-level antibiotic exposure
}

Erik Wistrand-Yuen (1) 1, Michael Knopp (10 1, Karin Hjort', Sanna Koskiniemi (1) 2, Otto G. Berg² \& Dan I. Andersson ${ }^{1}$

It has become increasingly clear that low levels of antibiotics present in many environments can select for resistant bacteria, yet the evolutionary pathways for resistance development during exposure to low amounts of antibiotics remain poorly defined. Here we show that Salmonella enterica exposed to sub-MIC levels of streptomycin evolved high-level resistance via novel mechanisms that are different from those observed during lethal selections. During lethal selection only rpsL mutations are found, whereas at sub-MIC selection resistance is generated by several small-effect resistance mutations that combined confer high-level resistance via three different mechanisms: (i) alteration of the ribosomal RNA target (gidB mutations), (ii) reduction in aminoglycoside uptake ( $c y \circ B, n u o G$, and trkH mutations), and (iii) induction of the aminoglycoside-modifying enzyme AadA (znuA mutations). These results demonstrate how the strength of the selective pressure influences evolutionary trajectories and that even weak selective pressures can cause evolution of high-level resistance.

\footnotetext{
${ }^{1}$ Department of Medical Biochemistry and Microbiology, Uppsala University, 75237 Uppsala, Sweden. ${ }^{2}$ Department of Cell and Molecular Biology, Uppsala University, 75237 Uppsala, Sweden. Correspondence and requests for materials should be addressed to D.I.A. (email: dan.andersson@imbim.uu.se)
} 
rrespective of whether antibiotics are used to treat infections in humans or animals, for growth promotion in animals, aquaculture, or plant production, a substantial fraction of these antibiotics will ultimately end up in the environment ${ }^{1}$. Thus, there are many environments such as wastewater, sludge, soil, and river water where bacteria are exposed for long periods of time to low concentrations of polluting antibiotics that are present because of anthropogenic influences ${ }^{2-7}$. Furthermore, low antibiotic concentrations (below the minimal inhibitory concentration, MIC) might be present in certain human/animal body compartments and tissues during therapeutic or growth promotion use. Previous studies showed that low levels of antibiotics (sub-MIC) can enrich for pre-existing resistant mutants in a bacterial population, indicating that certain antibiotics, disinfectants, and heavy metals could contribute to resistance evolution at concentrations that are several hundred-fold below the $\mathrm{MIC}^{8-13}$.

While many studies have examined the genetics of mutational antibiotic resistance selected at high levels (>MIC) of antibiotics, less is known about the effects of long-term exposure to low levels $(<\mathrm{MIC})$ of antibiotics ${ }^{14-19}$. When susceptible bacteria are exposed to antibiotic concentrations above the MIC they will die or stop growing, and only bacteria where resistance mutations were present prior to antibiotic exposure will be able to grow. In contrast, at sub-MIC concentrations of antibiotics the bacteria can still grow while they are under selection, generating a potentially different trajectory of evolution with progressive increase in resistance through the step-wise accumulation of resistance mutations with individually smaller effects. During selection at high concentrations of streptomycin the most common resistance mutations are target alteration mutations in the gene rpsL, encoding ribosomal protein $\mathrm{S} 12^{20,21}$, that confer highlevel resistance $>1024 \mathrm{mg} \mathrm{L}^{-122,23}$. Mutations in the electron transport chain that confer a small colony variant (SCV) phenotype can also cause a moderate increase in resistance (16-96 $\left.\mathrm{mg} \mathrm{L}^{-1}\right)$, which is often linked to a severe reduction in growth rate $24-26$. In this work, we investigated the evolution and genetics of antibiotic resistance selected during long-term exposure to streptomycin at sub-MIC level of the drug in Salmonella enterica, an important bacterial pathogen and model system for studies of antibiotic resistance. Results show that resistance evolution below the MIC of streptomycin occurs via different mechanisms compared to lethal selection and that high-level resistance can evolve also when bacteria are exposed to low antibiotic concentrations (sub-MIC).

\section{Results}

Mutant selection above MIC. Many previous studies have shown that $r p s L$ mutants are the major type of mutants found at selection above the $\mathrm{MIC}^{22,23}$. We reconfirmed these results and showed that when 10 independent cultures of susceptible Salmonella enterica serovar Typhimurium LT2 strain (designated S. typhimurium throughout the text) were selected on Mueller-Hinton (MH) agar for streptomycin resistance at $200 \mathrm{mg} \mathrm{L}^{-1}$ of streptomycin (50× above the MIC), 10/10 mutants had mutations in rpsL (amino acid substitutions: six K42R, one $\mathrm{K} 42 \mathrm{~N}$, one $\mathrm{K} 42 \mathrm{~T}$, and two K87R) that conferred the resistance. Whole-genome sequencing of six independent isolates confirmed that mutants selected on high streptomycin concentrations on agar plates had only rpsL mutations. Furthermore, we also performed a serial passage experiment (100 generations) in liquid MH containing $200 \mathrm{mg} \mathrm{L}^{-1}$ streptomycin. Whole-genome sequencing of five populations showed that the only resistance conferring mutations present in them were $r p s L$ mutations
(K42R). Thus, for 11 independent selections at high streptomycin levels only rpsL mutants were selected.

Mutant selection below MIC. To study evolution of antibiotic resistance in a susceptible bacterial population below MIC, 20 independent lineages of the streptomycin susceptible wild-type $S$. typhimurium were serially passaged for 900 generations in $\mathrm{MH}$ medium containing $1 \mathrm{mg} \mathrm{L}^{-1}$ of streptomycin, corresponding to $1 / 4$ of the MIC of the susceptible wild type. The concentration of streptomycin used causes an approximately $3 \%$ reduction in competitive growth rate of the susceptible wild type and was chosen to provide a weak sub-MIC selection. This estimation was based on previous work ${ }^{8}$, where in a serial passage competition experiment $1 \mathrm{mg} \mathrm{L}^{-1}$ streptomycin balances the $3 \%$ fitness cost conferred by an rpsL (K42R) mutation. Serial passage occurred every $24 \mathrm{~h}$ by transfer of $1 \mu \mathrm{l}$ of overnight culture $\left(5 \times 10^{9}\right.$ cells/ $\mathrm{ml})$ to $1 \mathrm{ml}$ of culture medium, generating a bottleneck of $5 \times 10^{6}$ cells during transfer. After serial passage, bacteria were plated on $\mathrm{MH}$ agar plates with different concentrations of streptomycin (8, $16,32,64,96,128,192$, and $\left.256 \mathrm{mg} \mathrm{L}^{-1}\right)$ to estimate the frequency of cells with different resistance levels. The populations were heterogeneous with regard to resistance and several of the lineages contained subpopulations (approximately $0.1-1 \%$ of the cells) with high levels of resistance (MIC of streptomycin $>96 \mathrm{mg}$ $\left.\mathrm{L}^{-1}\right)$. Clones with increased resistance were single-colony isolated from six independent lineages, and these purified clones were further analyzed. The MICs of streptomycin varied between $64 \mathrm{mg} \mathrm{L}^{-1}$ and $>1024 \mathrm{mg} \mathrm{L}^{-1}$ depending on the mutant. Four clones (obtained from four different lineages) with the highest streptomycin resistances $\left(>192 \mathrm{mg} \mathrm{L}^{-1}\right)$ were investigated further. In control experiments, where bacteria were serially passaged for 1000 generations in the absence of streptomycin, no streptomycin-resistant mutants were found ${ }^{27}$.

The spectra of mutations at sub-MIC and >MIC selection differ. To identify the mutations responsible for the increased resistance to streptomycin, the four clones with the highest resistance levels were whole-genome sequenced, and their sequences were compared to that of the ancestral strain to identify potential point mutations and structural rearrangements (deletions, duplications, insertions, and inversions). The results showed that all four strains had mutator phenotypes, with roughly 100 different point mutations per genome (Supplementary Table 1). The mutator phenotypes are likely to be caused by mutations in the DNA mismatch repair system since the strains had mutations in the mutSLH genes ${ }^{28}$.

To identify which mutations contributed to the high-level streptomycin resistance, we first examined if there were mutations present in the serially passaged strains that have previously been implicated in resistance. As shown by our present data and previous studies ${ }^{22,23}$, during selection for streptomycin resistance at high levels of streptomycin, mainly mutations in the gene rpsL, encoding the $30 \mathrm{~S}$ ribosomal subunit protein S12, are found. However, none of the clones isolated here from serial passage at sub-MIC concentrations had rpsL mutations. Instead, all of them had loss-of-function mutations in the gidB gene, encoding a 16S rRNA methyltransferase that has been linked to low levels of streptomycin resistance in Salmonella and Mycobacterium tuberculosis ${ }^{25,29}$. In addition, the strain with the highest streptomycin resistance also carried a mutation in the gene $t r k H$ encoding a potassium symporter, a gene previously shown to be involved in aminoglycoside resistance in Escherichia coli $i^{15,30}$.

To elucidate which additional mutations might be involved in the high-level resistance, we identified genes that were mutated in 


\section{Table 1 Isolated resistant mutants}

\begin{tabular}{|c|c|c|c|}
\hline Strain & Genotype & $\begin{array}{l}\text { Streptomycin MIC } \\
\left(\mathrm{mg} \mathrm{L}^{-1}\right)\end{array}$ & Reference \\
\hline DA6192 & Wild type (parent) & 4 & Strain collecti \\
\hline DA23868 & $\begin{array}{l}\text { Mutant isolated by plating serial passage population on } 256 \mathrm{mg} \mathrm{L}^{-1} \text { streptomycin and } \\
\text { picking a single colony (for complete genotype, see Supplementary Table 1) }\end{array}$ & 512 & This study \\
\hline DA23869a & $\begin{array}{l}\text { Mutant isolated by plating serial passage population on } 256 \mathrm{mg} \mathrm{L}^{-1} \text { streptomycin and } \\
\text { picking a single colony (for complete genotype, see Supplementary Table 1) }\end{array}$ & $>1024$ & This study \\
\hline DA23872a & $\begin{array}{l}\text { Mutant isolated by plating serial passage population on } 192 \mathrm{mg} \mathrm{L}^{-1} \text { streptomycin and } \\
\text { picking a single colony (for complete genotype, see Supplementary Table 1) }\end{array}$ & 512 & This study \\
\hline DA23874 & $\begin{array}{l}\text { Mutant isolated by plating serial passage population on } 128 \mathrm{mg} \mathrm{L}^{-1} \text { streptomycin and } \\
\text { picking a single colony (for complete genotype, see Supplementary Table 1) }\end{array}$ & 192 & This study \\
\hline DA23877a & $\begin{array}{l}\text { Mutant isolated by plating serial passage population on } 96 \mathrm{mg} \mathrm{L}^{-1} \text { streptomycin and } \\
\text { picking a single colony (for complete genotype, see Supplementary Table 1) }\end{array}$ & 96 & This study \\
\hline DA23879a & $\begin{array}{l}\text { Mutant isolated by plating serial passage population on } 96 \mathrm{mg} \mathrm{L}^{-1} \text { streptomycin and } \\
\text { picking a single colony (for complete genotype, see Supplementary Table 1) }\end{array}$ & 64 & This study \\
\hline DA25308 & DA23868, aadA::cat & 4 & This study \\
\hline DA25310 & DA23869, aadA::cat & 16 & This study \\
\hline DA25312 & DA23872, aadA::cat & 8 & This study \\
\hline DA25314 & DA23874, aadA::cat & 8 & This study \\
\hline DA26068 & DA23868, relA::Tn10dTet & 512 & This study \\
\hline DA26070 & DA23869, relA::Tn10dTet & 384 & This study \\
\hline DA26072 & DA23872, relA::Tn10dTet & 128 & This study \\
\hline DA26074 & DA23874, relA::Tn10dTet & 128 & This study \\
\hline DA27220 & DA23868, relA::Tn10dTet, $\Delta$ spoT::cat & 192 & This study \\
\hline DA27222 & DA23869, relA:: Tn10dTet, $\Delta$ spoT::cat & 256 & This study \\
\hline DA27224 & DA23872, relA::Tn10dTet, $\Delta$ spoT::cat & 96 & This study \\
\hline DA27226 & DA23874, relA::Tn10dTet, $\Delta$ spoT::cat & 48 & This study \\
\hline
\end{tabular}

Streptomycin resistance of $S$. typhimurium clones isolated from serial passage and the effects of aadA and relA, spoT inactivation mutations on resistance. MIC values presented are the median values of 2-5 Etests from biological replicates

Note that the four whole-genome sequenced clones contained many mutations (see Supplementary Table 1 for list of all mutations)

more than one lineage with the assumption that repeated occurrence of mutations in a specific gene is likely to reflect a response to the antibiotic selection, rather than fixation of a random non-selected mutation (however, it should be noted that media adaptation mutations could also show repeated appearance in independent lineages, see below). Thus, besides the gidB and trkH mutations the strains with the highest streptomycin resistances (Table 1 and Supplementary Table 1) had also accumulated mutations in genes involved in the respiratory electron transport chain, such as nuoG and nuoE, encoding subunits of $\mathrm{NADH}$ dehydrogenase and $c y o B$, encoding the cytochrome o oxidase subunit I. Two of the strains also carried mutations in the $z n u A B$ genes encoding proteins involved in zinc uptake. This generated a list of five candidate mutations: gidB, $\operatorname{trkH}, n u o G, c y o B$, and $z n u A$ that were examined further.

Combination of five mutations generated high-level resistance. The MIC of streptomycin for the S. typhimurium strain (DA6192) used as a starting ancestral strain for the sub-MIC cycling was $4 \mathrm{mg} \mathrm{L}^{-1}$ and the isolated resistant mutants had MIC values ranging from 192 to $>1024 \mathrm{mg} \mathrm{L}^{-1}$ (Table 1). To examine how the putative resistance mutations contributed to the highest level of resistance $\left(>1024 \mathrm{mg} \mathrm{L}^{-1}\right)$, we reconstructed all possible combinations of the five candidate genes: $\operatorname{gidB}, c y o B, n u o G, \operatorname{trkH}$, and $z n u A$ (identified as described above), generating 32 different mutants (Fig. 1, Table 2). Of the five reconstructed single mutants only the mutation in the gene gidB conferred a significant increase in resistance to streptomycin. Thus, the loss-of-function mutation $\operatorname{gidB}\left(\mathrm{Q} 169^{\star}\right)$ (where ${ }^{\star}$ represents a stop codon) gave a MIC of $32 \mathrm{mg} \mathrm{L}^{-1}$ for streptomycin, similar to previously reported values ${ }^{25}$. In contrast, the other single mutants gave no $(\operatorname{trkH}), 1.5$-fold $(z n u A)$, or 2 -fold $(c y o B, n u o G)$ increases in resistance (Table 2 and Fig. 1). By combining several of the mutations generating all potential double, triple, quadruple, and quintuple mutants, a step-wise increase in resistance was observed, reaching up to $>1024 \mathrm{mg} \mathrm{L}^{-1}$. Thus, adding mutations in the respiratory chain to a $\operatorname{gid} B\left(\mathrm{Q} 169^{*}\right)$ genetic background increased the resistance further, where mutations in $c y o B$ (G283D) or nuoG (D264G) individually increased the MIC to $96 \mathrm{mg} \mathrm{L}^{-1}$, while the combination of both gave a MIC of $256 \mathrm{mg}$ $\mathrm{L}^{-1}$ (Table 2 and Fig. 1). Reconstruction of the selected $z n u A$ (S19fs) (where "fs" represents a frame shift) mutation in a genetic background of $\operatorname{gidB}\left(\mathrm{Q} 169^{\star}\right), c y o B(\mathrm{G} 283 \mathrm{D})$, and $n u o G(\mathrm{D} 264 \mathrm{G})$ increased the MIC even further to $768 \mathrm{mg} \mathrm{L}^{-1}$ (Table 2 and Fig. 1). Finally, the addition of the trkH (T99A) mutation increased the resistance even further to a MIC of $>1024 \mathrm{mg} \mathrm{L}^{-1}$. A comparison of the resistance levels of the single mutants with the higher-order mutants showed that there were strong epistatic interactions between the mutations to generate the high-level resistance (Fig. 2 and Supplementary Table 2). Thus, the expected additive resistance (without epistasis) is a 36-fold increase in MIC in the quintuple mutant compared to the wild type, while the observed increase was $>220$-fold (Fig. 2a).

Other types of mutations that were found in several of the sequenced clones were mutations in genes in the maltose operon (malT and malF) and genes encoding tRNAs. The mal mutations had no effect on resistance and are likely to be media adaptation mutations ${ }^{31,32}$. Similarly, mutations in genes involved in flagellar biosynthesis $(f \lg C, f l g K, f l i N \text {, and } f l i J)^{27}$ were also seen in $\mathrm{MH}$ media adapted Salmonella strains, whereas the role of the tRNA mutations is unknown.

The role of the aadA gene and the stringent response in resistance. Previous studies showed that activation of the cryptic aminoglycoside resistance gene aadA in $S$. typhimurium is ppGpp-dependent, and that upregulation of this gene results in increased resistance to streptomycin ${ }^{25}$. The aadA gene encodes an aminoglycoside adenylyl transferase which inactivates the drug 


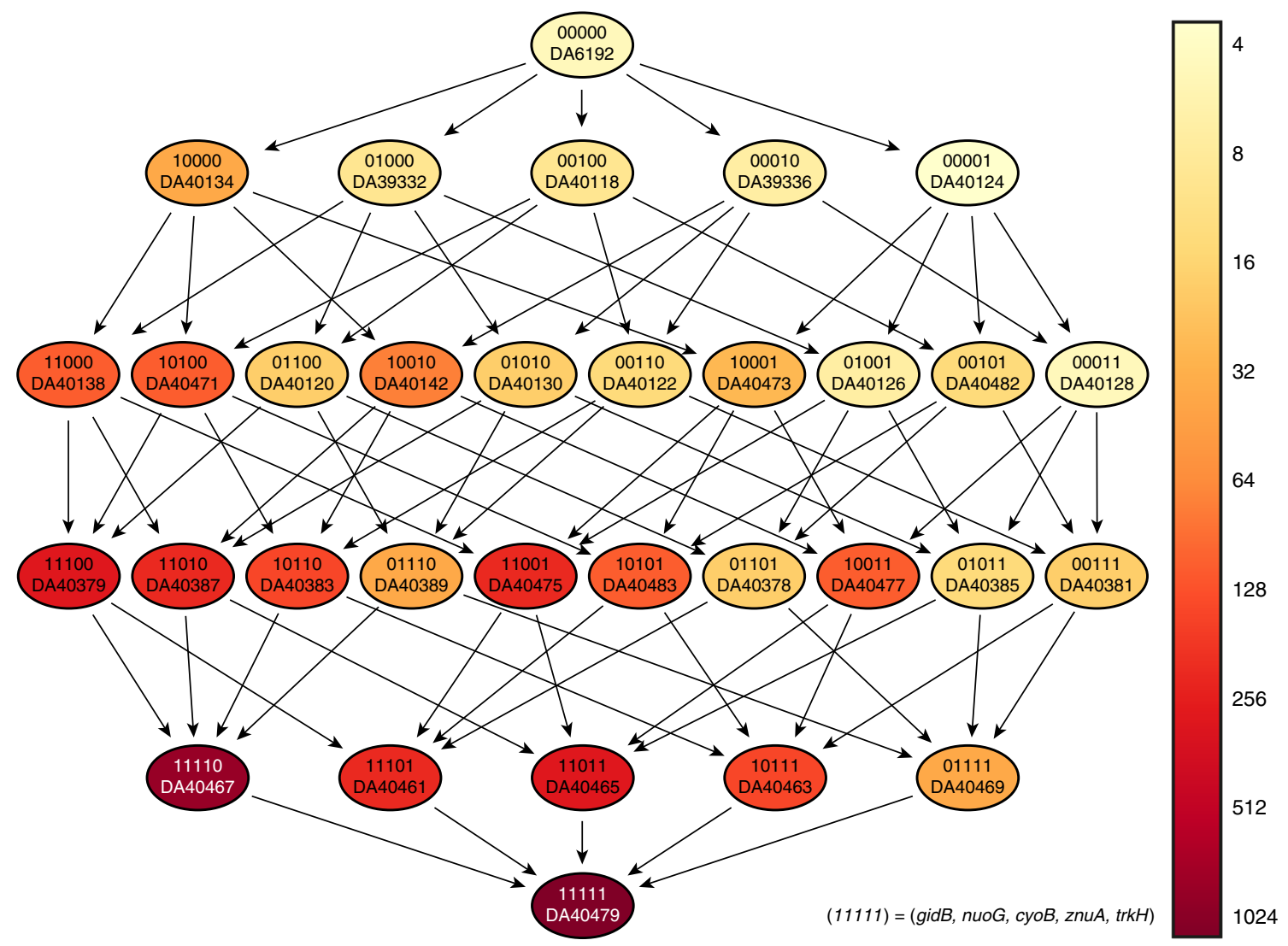

Fig. 1 The fitness landscape of streptomycin resistance. The color scale indicates the MIC of streptomycin in $\mathrm{mg} \mathrm{L}^{-1}$ for each reconstructed mutant. The genotype of each strain is written as a five-bit binary code, where 0 and 1 represent the wild-type and mutant sequences, respectively. See Table 2 for complete strain genotypes

by covalent addition of an adenylyl group. To examine the potential importance of aadA in the high-level streptomycin resistance observed in this study, we deleted the aadA gene in four of the clones isolated from the serial passage experiment (Table 1), as well as the reconstructed mutants (Table 2). This deletion resulted in a strong reduction of resistance for all mutants (Table 1), suggesting that the aadA gene has a central role in streptomycin resistance evolution at sub-MIC concentrations. Since the aadA gene is regulated by the stringent response, we also deleted the genes responsible for ppGpp synthesis, relA and spot, and this also lowered the resistance levels of the majority of the strains (Tables 1 and 2). These findings demonstrate that the high-level streptomycin resistance of the strains require the ability to induce stringent response and presence of the AadA protein.

Loss of $z n u A$ increase aadA transcript levels. Since high-level resistance requires the presence of the AadA protein (Tables 1 and 2), we determined if the increase in resistance in the reconstructed mutants was due to elevated aadA expression. We isolated total RNA at late exponential phase from all single mutants and two double, two triple, two quadruple, and the quintuple mutant and measured aadA transcript levels using qRT-PCR (Fig. 3). We observed a 7-fold increase in aadA expression in the $z n u A$ single mutant, while all other single mutants showed no significant increase. In accordance with this, all mutants with more than one mutation that also included $z n u A$ showed increased aadA expression levels to the same extent as the $z n u A$ single mutant. The two reconstructed clones containing multiple mutations without $z n u A$ (the double and triple mutants $\operatorname{gidB}, \operatorname{trkH}$, and $\operatorname{gidB}, n u o G, \operatorname{trk} H$ ) showed wild-type levels of $\operatorname{aad} A$ transcript. Thus, the $z n u A$ mutations (but not $\operatorname{gid} B, \operatorname{trkH}$, $c y o B$, or $n u o G$ mutations) confer increased streptomycin resistance by causing aadA upregulation and inactivation of streptomycin via adenylylation. To test whether loss of ZnuA rather than the production of a frameshifted protein is causing the observed phenotype we constructed a deletion of the $z n u A$ reading frame in a wild-type background as well as in a quadruple mutant consisting of gidB, nuoG, cyoB, and trkH (Fig. 3). In both cases the deletion of $z n u A$ resulted in a 11-fold upregulation of aadA transcript levels, showing that loss of $\mathrm{ZnuA}$ is responsible for the observed phenotype. In addition, the upregulation of aadA due to loss of ZnuA was completely restored if the cells were grown in the presence of excess zinc, demonstrating that it is zinc starvation and not lack of the ZnuA protein per se that caused upregulation of AadA.

cyoB, nuoG, and trkH mutations reduce streptomycin uptake. Since it is known that proton motive force is needed for streptomycin uptake $e^{33-36}$ and that the cyoB, nuoG, and trkH mutations are likely to impair electron transport ${ }^{30,37}$, we used radioactive streptomycin to measure if uptake was reduced in the mutants. Results show (Fig. 4) that one of the single mutants $(c y o B)$ and the triple mutant $(\operatorname{trkH}, n u o G, c y o B)$ had a significant reduction in intracellular levels of tritiated dihydrostreptomycin, supporting the notion that these mutations confer increased resistance by impairing drug uptake. While the observed decrease in intracellular streptomycin is only moderate, streptomycin uptake and resistance level does not necessarily correlate in a linear and proportional way, and a small effect on uptake could conceivably have a large effect on resistance. Additionally, the reduction in streptomycin uptake is likely to be underestimated 


\section{Table 2 Reconstructed strains}

\begin{tabular}{|c|c|c|c|c|c|c|c|c|}
\hline Strain & gidB & trkH & cyoB & nuoG & znuA & $\begin{array}{l}\text { Streptomycin MIC } \\
\left(\mathrm{mg} \mathrm{L}^{-1}\right)\end{array}$ & $\begin{array}{l}\text { Streptomycin MIC with } \\
\Delta \text { aadA }\end{array}$ & $\begin{array}{l}\text { Streptomycin MIC with relA: } \\
\text { Tn10 spoT:acat }\end{array}$ \\
\hline DA6192 & wt & wt & wt & wt & wt & 4 & 2 & 1.5 \\
\hline DA40134 & Q169* & & & & & 32 & 4 & 16 \\
\hline DA39332 & & & & D264G & & 8 & 3 & 6 \\
\hline DA40118 & & & G283D & & & 8 & 4 & 8 \\
\hline DA39336 & & & & & S19fs & 6 & 2 & 1.5 \\
\hline DA40124 & & T99A & & & & 3 & 2 & 2 \\
\hline DA40138 & Q169* & & & D264G & & 96 & 8 & 48 \\
\hline DA40471 & Q169* & & G283D & & & 96 & 12 & 48 \\
\hline DA40120 & & & G283D & D264G & & 16 & 8 & 16 \\
\hline DA40142 & Q169* & & & & S19fs & 64 & 4 & 24 \\
\hline DA40130 & & & & D264G & S19fs & 16 & 4 & 8 \\
\hline DA40122 & & & G283D & & S19fs & 12 & 6 & 6 \\
\hline DA40473 & Q169* & T99A & & & & 24 & 6 & 48 \\
\hline DA40126 & & T99A & & D264G & & 6 & 4 & 6 \\
\hline DA40482 & & T99A & G283D & & & 12 & 6 & 12 \\
\hline DA40128 & & T99A & & & S19fs & 4 & 2 & 3 \\
\hline DA40379 & Q169* & & G283D & D264G & & 256 & 16 & 96 \\
\hline DA40387 & Q169* & & & D264G & S19fs & 192 & 8 & 96 \\
\hline DA40383 & Q169* & & G283D & & S19fs & 128 & 8 & 96 \\
\hline DA40389 & & & G283D & D264G & S19fs & 32 & 12 & 16 \\
\hline DA40475 & Q169* & T99A & & D264G & & 192 & 12 & 64 \\
\hline DA40483 & Q169* & T99A & G283D & & & 96 & 16 & 48 \\
\hline DA40378 & & T99A & G283D & D264G & & 16 & 12 & 16 \\
\hline DA40477 & Q169* & T99A & & & S19fs & 96 & 4 & 24 \\
\hline DA40385 & & T99A & & D264G & S19fs & 12 & 4 & 12 \\
\hline DA40381 & & T99A & G283D & & S19fs & 16 & 6 & 12 \\
\hline DA40467 & Q169* & & G283D & D264G & S19fs & 768 & 24 & 256 \\
\hline DA40461 & Q169* & T99A & G283D & D264G & & 192 & 24 & 96 \\
\hline DA40465 & Q169* & T99A & & D264G & S19fs & 256 & 8 & 96 \\
\hline DA40463 & Q169* & T99A & G283D & & S19fs & 128 & 12 & 128 \\
\hline DA40469 & & T99A & G283D & D264G & S19fs & 32 & 6 & 12 \\
\hline DA40479 & Q169* & T99A & G283D & D264G & S19fs & 1024 & 16 & 256 \\
\hline
\end{tabular}

Genotypes and streptomycin resistances of reconstructed strains and the effects of aadA and relA, spoT inactivation on resistance. The symbol "*" represents a stop codon, while "fs" represents a frame shift mutation. MIC values presented are the median values of 2-5 Etests from biological replicates

since the background signal in all strains might partly result from extracellularly bound streptomycin.

Modeling the step-wise selection of five mutations. To examine the dynamics of the step-wise enrichment of several mutations in one clone and determine under which conditions five step-wise mutations could accumulate in 900 generations under this weak selection, we modeled the selection process (for details, see Methods). In these calculations, we applied the experimentally determined population sizes and generations of growth used during serial passage. We also assumed that all selected mutations contribute independently to resistance/fitness and that each mutation has the same selection coefficient $s=0.006$, such that five of them together give a $3 \%$ growth advantage in the presence of the antibiotic (during the selection the used concentration of streptomycin at $1 / 4$ MIC reduced the growth rate of the susceptible wild type by $3 \%)$. Furthermore, a mutator mutation is assumed to occur with the same rate, $u$, as each of the five selected resistance mutations (which are all loss-of-function mutations). In addition, it is assumed (as also observed experimentally) that the highlevel-resistant mutants reached a frequency of $1 \%$ in the evolving population after 900 generations of growth. Results show that without mutators, $1 \%$ penetration after 900 generations can be achieved only with an unrealistically high mutation rate, $u=10^{-3}$, for each individual resistance mutation. In contrast, when mutators with mutation rate $v$ are present, the mutant with five resistance mutations can reach $1 \%$ penetration in 900 generations within a realistic parameter range where mutation rate $u$ varies ca. 10 -fold between $5 \times 10^{-7}$ and $4 \times 10^{-6}$ and the ratio $v / u$ also varies 10 -fold between ca. $10^{2}$ and $10^{3}$ (Table 3, Fig. 5). Thus, even with a weak selection $(s=0.03)$ and only 900 generations of evolution, it is possible to step-wise accumulate five resistance mutations provided mutator clones appear early during serial passage.

\section{Discussion}

In this study, we exposed susceptible wild-type bacteria to constant low levels of antibiotics (4-fold below the MIC, $1 \mathrm{mg} \mathrm{L}^{-1}$ ) for 900 generations, and found that high-level resistance (up to $>1024 \mathrm{mg} \mathrm{L}^{-1}$ ) evolved in several independent lineages. By whole-genome sequencing and genetic reconstructions, we identified five causative resistance mutations of individual clones. The mutations had individually small effects, but when combined in a wild-type background they resulted in high-level resistance due to strong positive epistasis between certain mutations (Fig. 2, Supplementary Table 2). Since the evolved strains contained more mutations than the five that we studied in detail it is possible that other mutations also contributed to the resistance and fitness phenotypes.

An important question is how consecutive mutations in five different genes can accumulate over only 900 generations of selection with the weak selection used. Our modeling results show that with a $3 \%$ reduction in growth rate, a mutation rate for each consecutive mutation of around $3.5-5 \times 10^{-4}$ per cell per generation is required for the step-wise accumulation of five consecutive mutations in 900 generations with the serial passage 
a

300

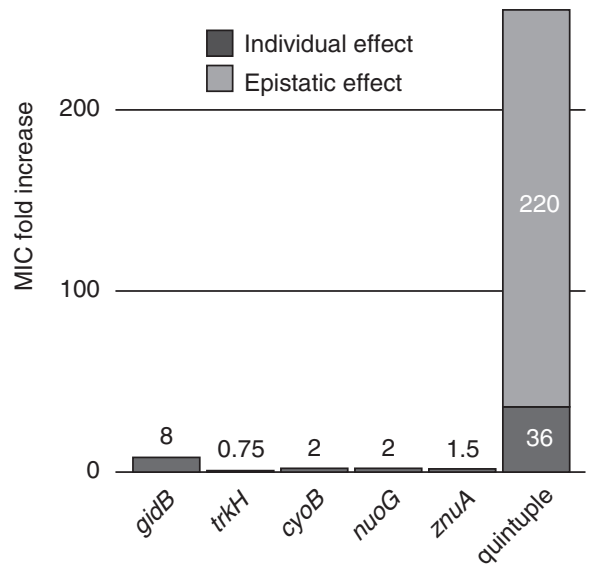

b

3

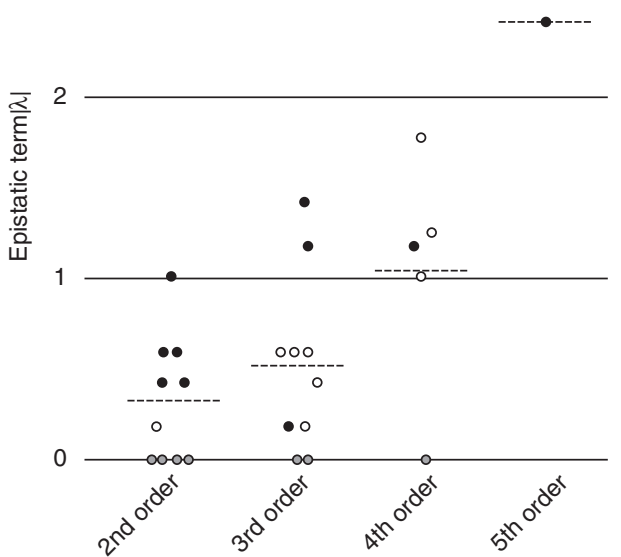

Fig. 2 Epistatic interactions. a Five mutations generate higher resistance than expected from their individual effects. The MIC increase based on individual additive effects is indicated in dark gray, while fold increase based on epistatic interactions is indicated in light gray. b The epistatic interactions increase with higher orders. Positive epistasis (filled circles) predominates at the 2 nd and 5th order, while negative epistasis (empty circles) predominates at 3rd and 4 th order. The strongest epistasis is observed in the quintuple mutant. Gray circles represent no epistatic interactions. The full dataset of epistatic interactions can be found in Supplementary Table 2

population sizes used and when the quintuple mutant constitutes $1 \%$ of the total population. All the resistance phenotypes are caused by loss-of-function mutations in the five different target genes and from previous work it is known that the average lossof-function rate in a gene in a wild-type E. coli/S. enterica is approximately $10^{-6}$ per cell per generation ${ }^{38,39}$. In addition, all the examined clones are mutators, and the likely explanation for the mutator phenotype is that step-wise selection of several independent resistance mutations enriches for the mutators. It is well described that mutator alleles can rapidly become enriched in asexual populations during step-wise adaptation by second-order selection because they remain associated with the rare favorable resistance mutations they generate ${ }^{14,40-43}$. Importantly, the mutators (MutHLS mismatch repair system) found in this study exhibit an approximately 150 -fold increase in mutation rate as compared to the wild type (Supplementary Table 3), which brings the expected loss-of-function for each individual gene to $10^{-4}$ per cell per generation, which is near the theoretically required mutation rate. We also attempted to experimentally determine which of the many potential routes to the high-level-resistant quintuple mutant (Fig. 1) occurred in the different lineages and how fitness changed in the evolving populations over time. However, this was not possible due to the fact that all of these strains are mutators and as a result the heterogeneity in each population is very high, making a time series analysis by deep sequencing of the evolving population difficult. In addition, the high heterogeneity in combination with the mutator phenotype also preclude us from making accurate measurements of small fitness differences $(<1 \%)$ by competition since the competitor strains would rapidly acquire media adaptation mutations that alter their fitness.

How is resistance conferred by the identified mutations? Based on the experiments presented here and previous studies, we propose that the high-level resistance is caused by the combined effect of three different mechanisms: (i) rRNA target alteration (due to gidB mutations), (ii) reduced aminoglycoside uptake (due to $c y o B, n u o G$, and $\operatorname{trkH}$ mutations), and (iii) induction of the aminoglycoside modifying enzyme AadA (due to znuA mutations) (Fig. 6). With regard to the first mechanism, all of the strains had loss-of-function mutations in the gidB gene, encoding a 16S rRNA methyltransferase. This enzyme methylates the 530 loop of 16S rRNA and if the modification is absent streptomycin binding to the ribosome is reduced ${ }^{29}$. Mutations in this gene have been reported to confer a moderate increase resistance to streptomycin in several species of bacteria, including S. enterica ${ }^{25}$ and M. tuberculosis ${ }^{29}$.

The second mechanism includes mutations that are located in genes encoding proteins involved in the electron transport chain, such as genes encoding subunits of $\mathrm{NADH}$ dehydrogenase (nuoG) and cytochrome o oxidase (cyoB). Aminoglycosides are cationic molecules, and they are believed to first penetrate the outer membrane through porins, and then cross the cytoplasmic membrane in a process that is dependent on electron transport through quinones and a sufficiently high membrane potential $^{35,36}$. The mutations we observed in the respiratory chain $(c y o B$ and $n u o G)$ could potentially reduce electron transport and the membrane potential, and as result reduce streptomycin uptake. Similar mutations have also previously been found in streptomycin-resistant SCVs of Salmonella ${ }^{24,25}$, but while the SCVs have a very slow growing phenotype, the mutants selected in this study at sub-MIC levels of streptomycin have higher growth rates (Supplementary Table 3). Besides genes involved directly in the respiratory chain, the mutant with the highest level of streptomycin resistance also had a mutation in the potassium symporter TrkH. The gene encoding TrkH has previously been linked to streptomycin resistance, probably by affecting the membrane electrochemical potential ${ }^{30,37}$. Uptake assays with radiolabeled dihydrostreptomycin showed that, as expected, the $c y o B, n u o G$, and $\operatorname{trkH}$ mutants were impaired in drug accumulation, providing a conceivable explanation for how these mutations confer increased resistance (Fig. 4).

The third mechanism of resistance, enzymatic modification of streptomycin, is dependent on the expression of the cryptic aminoglycoside resistance gene aadA. Previous studies of Salmonella SCVs have shown that the aadA gene can be upregulated through the stringent response ${ }^{25}$, a stress response system that uses the global regulator ppGpp to control the distribution of resources between synthesis of ribosomes when nutrients are abundant, and the increased synthesis of metabolic enzymes during amino acid starvation ${ }^{44}$. Deleting the aadA gene in the 


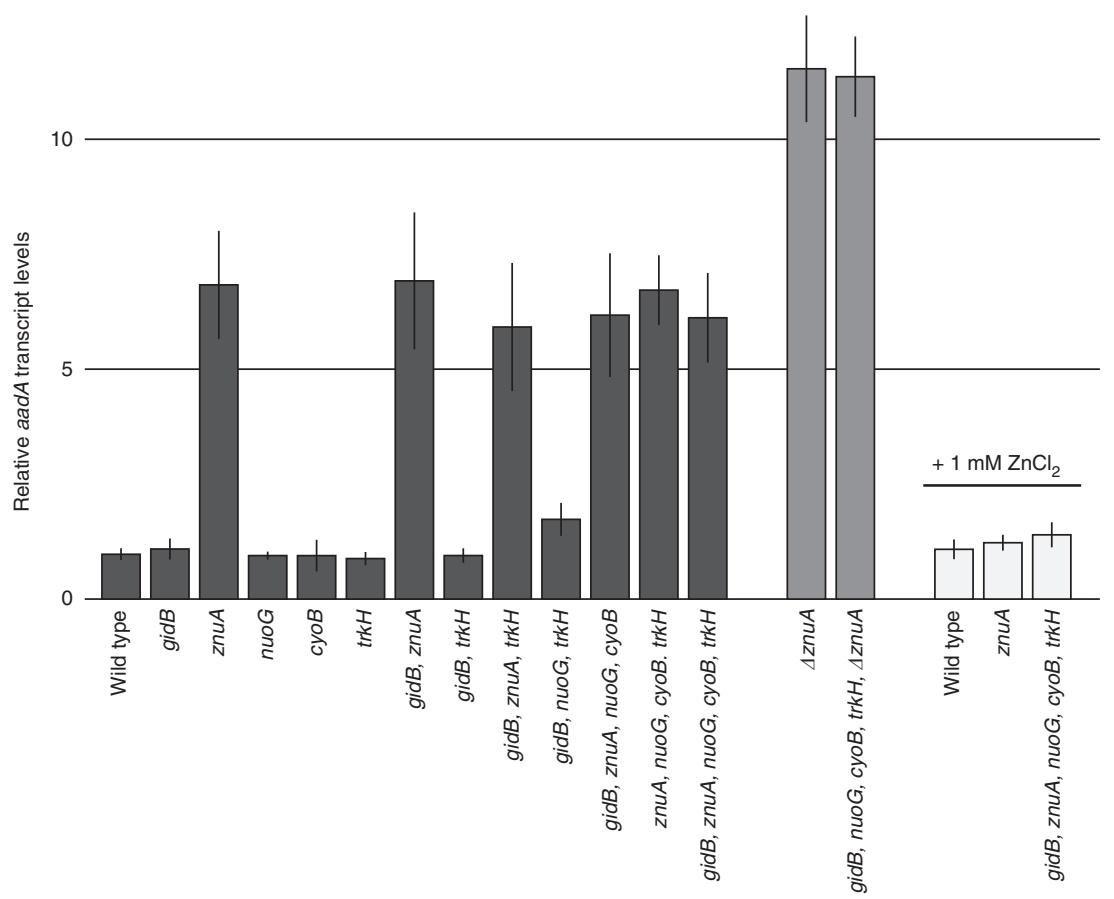

Fig. 3 Relative aadA transcript levels. Transcript levels of the aadA gene were determined in reconstructed mutants (dark gray), znuA deletion mutants (gray), and reconstructed mutants grown in the presence of $1 \mathrm{mM} \mathrm{ZnCl}$ (white). Transcript levels were normalized to the housekeeping genes cys $G$ and hcaT and all values are relative to transcript levels in the wild type. The error bars represent the standard deviation of two biological and three technical replicates each

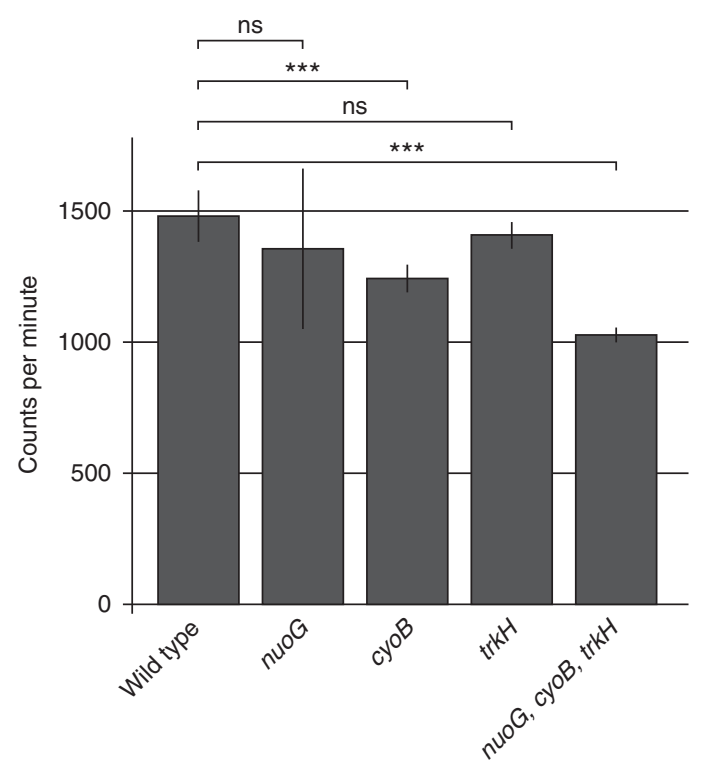

Fig. 4 Intracellular uptake of H3-dihydrostreptomycin. Cells from late exponential phase were incubated $30 \mathrm{~min}$ in presence of $50 \mathrm{nCi} \mathrm{ml}^{-1}$ tritiated dihydrostreptomycin and washed to remove extracellular $\mathrm{H} 3-$ dihydrostreptomycin. Counts per minute were determined for two biological and two technical replicates each. The error bars represent standard deviation. $t$-tests were performed to calculate the significance of the differences between mutants and wild type, where ns (not significant) indicates a $p$-value over 0.05 and three asterisks indicate high significance with $p$-values $<0.01$

mutants (both original and reconstructed) lowered resistance by 1.5 -fold to 100-fold (Tables 1 and 2). However, after deletion of relA and spoT, which removes any ppGpp-dependent regulation $^{45}$, strains carrying mutations in gidB, cyoB, nuoG, and/or
trkH still show increased resistance to streptomycin compared to a relA, spoT-deficient wild-type strain indicating that their effect on resistance is not dependent on stringent response but on target modification via gidB and reduced uptake via $c y o B, n u o G$, and trkH. Only the effect of $z n u A$ mutations seemed to be abolished in a $\operatorname{rel} A$, spoT-deficient background (Table 2). In addition, we observed that the mutation in $z n u A$ alone was responsible for elevated aadA expression levels (Fig. 3). The periplasmic zinc binding protein $\mathrm{ZnuA}$ is part of the ZnuABC system. This system is of particular importance under zinc-limiting conditions, ensuring the high-affinity uptake of $\mathrm{Zn}^{2+46}$. Despite the fact that this system is repressed in the presence of adequate concentrations of zinc ${ }^{47}$ it is likely that basal levels are still expressed even during growth in rich medium like $\mathrm{MH}$ broth. Loss-of-function mutations in $z n u A$ are therefore likely to disrupt zinc homeostasis, an ion with a central role in essential cellular enzymes and processes $^{48}$, and cause activation of cellular responses that subsequently could alter gene expression patterns. To determine if the observed upregulation of aadA in $z n u A$-deficient mutants is mediated via increased ppGpp levels we measured the transcript levels of iraP, which is known to be positively regulated by elevated ppGpp levels ${ }^{49}$, in a subset of mutants. None of the tested strains showed an increase in iraP transcript levels indicating a ppGpp-independent mechanism even for znuA mutants. Furthermore, ppGpp levels were not consistently increased in the $z n u A$ mutants (Supplementary Fig. 1). The transcriptional changes caused by ppGpp are often augmented by the transcription factor DksA ${ }^{50-52}$. Since this protein contains a central Cys4 Zn-finger motif that is likely to be essential for correct folding and functioning ${ }^{53,54}$, we hypothesized that the observed effect of zinc depletion on aadA upregulation might be caused by the loss of functional DksA. However, deletion of $d k s A$ in a wildtype background did not cause upregulation of aadA. Additionally, the introduction of a zinc-independent $d k s A$ paralog from Pseudomonas aeruginosa $a^{55}$ in a $z n u A$ mutant did not restore wild-type levels (Supplementary Fig. 2), showing that misfolding 
Table 3 Penetration of five mutations $\mathrm{Fo}_{5}+\mathrm{Fm}_{5}$ after 900 generations

\begin{tabular}{|c|c|c|c|c|c|c|c|}
\hline$v / u$ & 90 & 125 & 203 & 285 & 400 & 460 & 1055 \\
\hline \multicolumn{8}{|l|}{$u$} \\
\hline $5 \times 10^{-7}$ & $5 \times 10^{-8}$ & $2.5 \times 10^{-7}$ & $2.8 \times 10^{-6}$ & $1.5 \times 10^{-5}$ & $8.3 \times 10^{-5}$ & $1.7 \times 10^{-4}$ & 0.010 \\
\hline $2 \times 10^{-6}$ & $1.9 \times 10^{-4}$ & $9.5 \times 10^{-4}$ & 0.010 & 0.048 & 0.17 & 0.24 & 0.65 \\
\hline $3 \times 10^{-6}$ & 0.002 & 0.010 & 0.083 & 0.24 & 0.43 & 0.5 & 0.76 \\
\hline $4 \times 10^{-6}$ & 0.010 & 0.045 & 0.23 & 0.42 & 0.57 & 0.62 & 0.81 \\
\hline
\end{tabular}

of DksA under zinc starvation is not responsible for the observed phenotypes. While the exact mechanism of aadA upregulation via $z n u A$-mediated zinc-depletion remains unclear, our results suggest that it is not mediated via ppGpp or DksA, but requires functional RelA and SpoT, possibly due to a dominant regulatory effect exerted by these proteins.

What are the general implications of this work? First, and most importantly, our results demonstrate that very weak and constant selective pressures of antibiotics can result in the evolution of high-level resistance. Thus, an implication from these results is that there is no necessary correlation between strength of selection and level of resistance evolved, and as a consequence it is conceivable that the low antibiotic levels present in many environments could drive the evolution of clinically relevant high-level resistance. Second, our data show that mutational spectra can differ strongly between different selective strengths. Thus, at lethal selections only rpsL mutations are found, whereas at subMIC selections a different set of mutations are selected. A likely explanation for this difference is that at $>$ MIC levels of drug only rpsL mutations provide a sufficiently high level of resistance to be enriched, whereas at low concentrations the more common mutations in the genes identified here are preferentially observed (even though rpsL mutations occur but at a much lower rate). Third, these results show that the high-level resistance can result from many different mutations of small individual effect that show positive epistasis. Thus, among the five genes examined only mutations in the gidB gene conferred measurable resistance by itself, whereas when the five mutant genes were combined resistance reached very high levels. Furthermore, a majority of these mutations were in genes that are not typical resistance genes (i.e., $c y o B, n u o G, \operatorname{trkH}$, and $z n u A$ ). This finding raises the question if a substantial "dark matter" of unknown resistance mutations for other classes of antibiotics exists in clinical isolates that apart from the generally easily identified bona fide resistance genes/mutations may be involved in resistance evolution and make a significant contribution to the clinical resistance phenotype $\mathrm{i}^{14,18,19,56}$. Thus, it is conceivable that the initial selection for common mutations of small effect could act as a stepping stone for the subsequent occurrence of a rarer large effect genetic event (e.g., horizontal gene transfer of a bona fide resistance gene) and thereby act as a driver of resistance evolution. Also, considering that individually the mutations in $c y o B, n u o G, \operatorname{trk} H$, and $z n u A$ conferred no/minor increases in resistance, these types of atypical resistance mutations would be difficult to identify in clinical isolates using standard genetic procedures and reconstruction. That is, transfer of DNA from a resistant strain by transformation/transduction to a susceptible strain to identify potential resistance mutations is unlikely to work since the individual mutations except for gidB had minor effects on resistance. Instead, the clinical identification of these types of mutations would first require laboratory selection and reconstitution experiments (as done here) for identification, and then a subsequent comparative analysis of susceptible and resistant clinical isolates to search for their potential presence in resistant isolates. Finally, it is worthwhile noting that the potential occurrence of atypical resistance mutations in clinical isolates would make sequence-based in silico predictions of antibiotic resistance more challenging.

\section{Methods}

Strains and growth conditions. All strains used in this study were derived from Salmonella enterica serovar Typhimurium LT2 (designated S. typhimurium in the text) and are listed in Tables 1 and 2, and Supplementary Tables 4 and 5. The liquid and solid media used for bacterial growth were MH broth (Becton Dickinson, MD, USA), $\mathrm{MH}$ agar ( $\mathrm{MH}$ broth supplemented with $1.5 \%$ agar), and Luria-Bertani (LB) agar (Sigma-Aldrich, MO, USA). Strains were grown at $37^{\circ} \mathrm{C}$, and liquid cultures were aerated by shaking.

Selection for resistance above MIC. Selection of $S$. typhimurium mutants with decreased susceptibility to streptomycin was performed by plating 10 independent cultures (each inoculated with $10^{3} \mathrm{cfu}$ ) of the wild-type strain DA6192 on $\mathrm{MH}$ agar containing $200 \mathrm{mg} \mathrm{L}^{-1}$ streptomycin (Sigma-Aldrich). Approximately $10^{10} \mathrm{cfu}$ from each culture were plated. The mutants (one per independent culture) were randomly picked after $24 \mathrm{~h}$ of incubation at $37^{\circ} \mathrm{C}$ and re-isolated on plates with the same concentration of streptomycin. Cultures of isolated clones were grown over night at $37^{\circ} \mathrm{C}$ in $\mathrm{MH}$ broth without selection and frozen in $-80^{\circ} \mathrm{C}$ with $10 \%$ sterile DMSO. The rpsL gene in the mutants was PCR amplified with primers binding upstream and downstream of the coding region using DreamTaq DNA polymerase (Thermo Scientific). Populations of S. typhimurium were also evolved in presence of high levels of streptomycin in liquid medium by passaging 10 lineages of the wild-type strain DA6192 in $\mathrm{MH}$ medium with $200 \mathrm{mg} \mathrm{L}^{-1}$ streptomycin. The lineages were started from overnight cultures of independent colonies grown in $\mathrm{MH}$ without selection. The evolution experiment was initiated with an inoculum size of approximately $10^{10}$ cells. The cultures were serially passaged by 1000 -fold dilutions in $1 \mathrm{ml}$ batch cultures every $24 \mathrm{~h}$, and after 100 generations of cycling the cultures were frozen in $-80^{\circ} \mathrm{C}$ with $10 \%$ sterile DMSO. Six mutants selected on solid media and five populations evolved in liquid media were whole-genome sequenced to identify possible streptomycin resistance mutations outside of $r p s L$ (Supplementary Table 4).

Laboratory evolution of resistance at sub-MIC. The evolution of de novo resistance has been described previously ${ }^{8}$. Briefly, bacteria were grown in $\mathrm{MH}$ medium with $1 \mathrm{mg} \mathrm{L}^{-1}$ of streptomycin for $24 \mathrm{~h}$ at $37^{\circ} \mathrm{C}$ with shaking and then 1 $\mu \mathrm{l}$ culture was transferred generating a maximum and minimum population of $5 \times$ $10^{9} \mathrm{ml}^{-1}$ and $5 \times 10^{6} \mathrm{ml}^{-1}$, respectively, during each step of the serial passage. After 900 generations (approximately 90 serial passages) of cycling, $10^{5}$ cells were plated onto LB agar containing different concentrations of streptomycin $(8,16,32$ $64,96,128,192$, and $256 \mathrm{mg} \mathrm{L}^{-1}$ ), and 2-3 resistant clones were isolated from each lineage where they represented a fraction of the population of approximately $1 \%$ (Supplementary Table 5). The isolated clones were frozen in $-80^{\circ} \mathrm{C}$ in $10 \%$ DMSO, and the evolving lineages were frozen every 100 generations.

Whole-genome sequencing. Genomic DNA (gDNA) was extracted from $4 \mathrm{ml}$ of overnight cultures of the mutants isolated after 900 generations of growth in $1 \mathrm{mg} \mathrm{L}^{-1}$ of streptomycin using the Genomic-tip 100/G columns and the Genomic DNA Buffer Set (Qiagen, The Netherlands), according to the manufacturer's instructions. Quality of gDNA were examined using NanoDrop spectrophotometer (Thermo Scientific, Waltham, MA, USA) and separated by electrophoresis on $0.8 \%$ (w/v) agarose gels for visualization. The gDNA was sent to the Beijing Genomic Institute sequencing facility (Hong Kong, China) for whole-genome resequencing using the Illumina HiSeq 2000 sequencing system (Illumina Inc., San Diego, CA, USA) with $50 \times$ coverage. Briefly, sequencing libraries per gDNA were prepared according to the Solexa sequencing protocols (Illumina Inc., San Diego, CA, USA). 

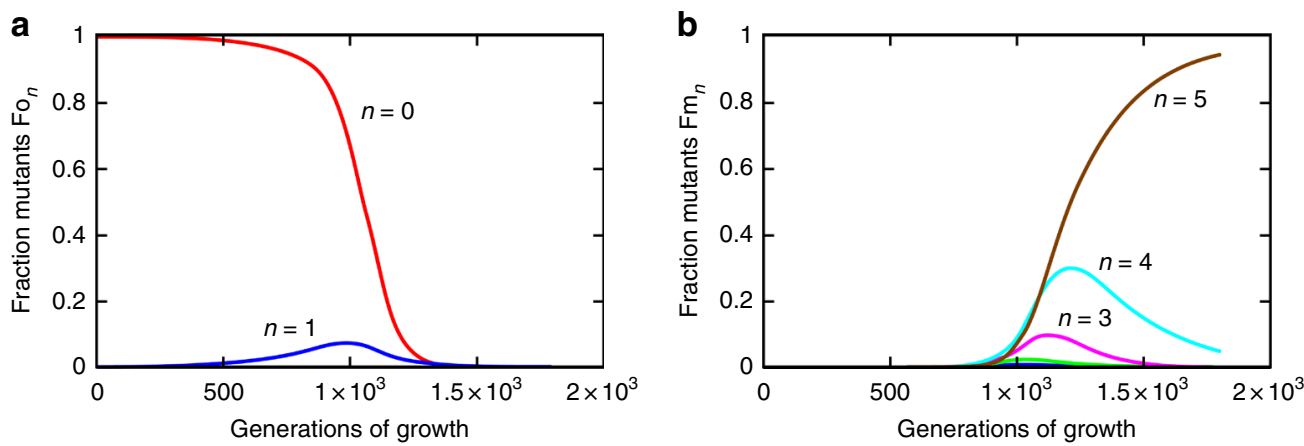

Fig. 5 Population dynamics of different mutant types. Time development of the population fractions for $u=2 \times 10^{-6}$ and $v / u=200$. The fractions of the different variants over time in generations are shown on the $y$-axis, where $\mathrm{Fo}_{n}$ and $\mathrm{Fm}_{n}$ are the fractions of cells with $n$ selected mutations for non-mutators and mutators, respectively. Non-mutators (a) contribute almost exclusively to $n=0$ (red curve) and $n=1$ (blue curve), while mutators (b) dominate totally in the fractions that carry three or more mutations; in the group with $n=2$ (green curve), there is some mixing between mutators and non-mutators

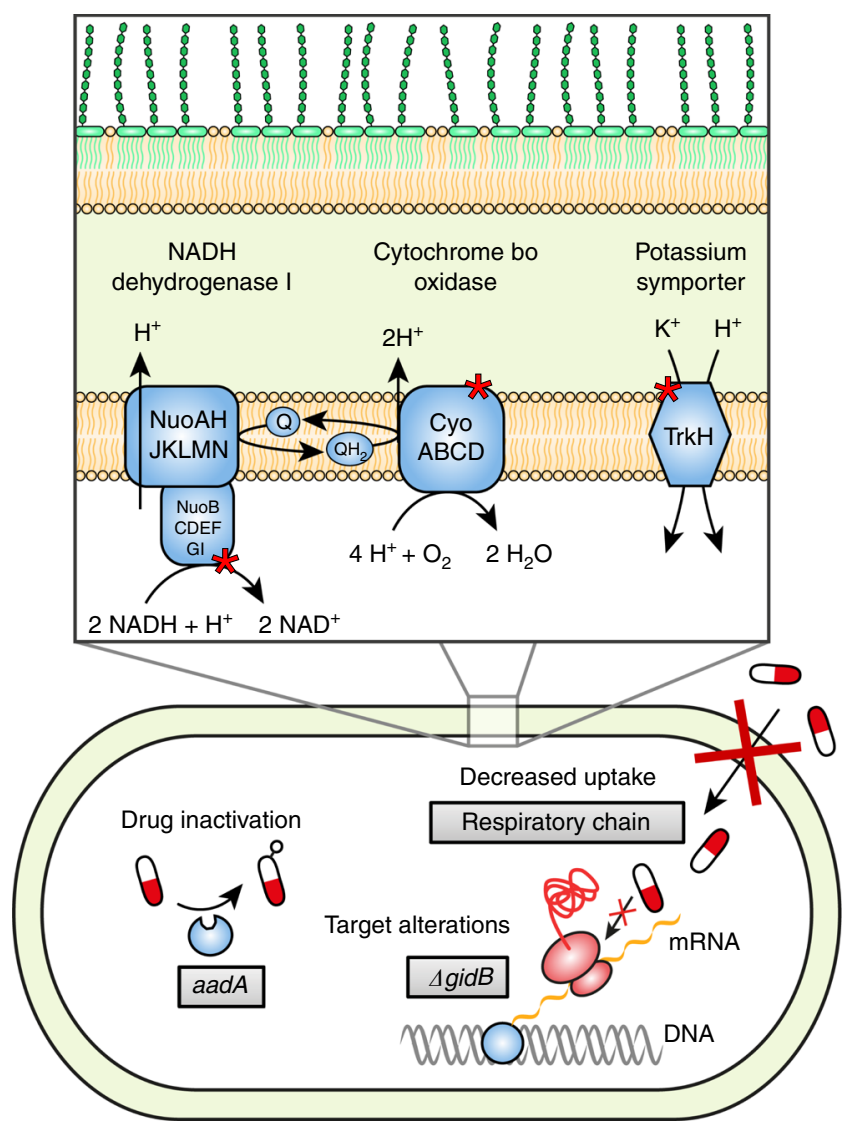

Fig. 6 Three different resistance mechanisms that act in synergy. Increased expression of the aminoglycoside adenyl transferase gene aadA via inactivation of the gene znuA lowers the concentration of active drug through chemical modification, mutations in gidB indirectly modifies the drug target to decrease binding, and mutations in the respiratory chain lowers the membrane potential, causing a decrease in uptake of the drug

Illumina analysis pipeline was used for image analysis, base calling, and quality score calibration. Subsequently, raw sequence reads were quality filtered and exported as FASTQ files resulting in 12,000,000 high-quality sequence reads per gDNA isolated. For the clones and populations selected with $200 \mathrm{mg} \mathrm{L}^{-1}$ of streptomycin, DNA extraction was done from $1 \mathrm{ml}$ of overnight cultures using Epicentre MasterPure Complete DNA and RNA Purification Kit (Illumina Inc., San Diego, CA, USA) following the manufacturer's instructions. Libraries to be sequenced with Miseq $(2 \times 300)$ were made using the Illumina's Nextera XT kit, and samples were dual-indexed and pooled. The paired-end sequence reads have been deposited to the NCBI Sequence Read Archive (SRA) with accession no. SRP133288.

The reads were mapped to the previously sequenced S. typhimurium DA6192 in house reference genome ${ }^{27}$ using CLC Genomics Workbench version 11 (Qiagen Bioinformatics, Aarhus, Denmark) with default parameters (masking mode $=$ no masking; match score $=1$; mismatch cost $=2$; cost of insertions and deletions $=$ linear gap cost; insertion cost $=3$; deletion cost $=3$; length fraction $=0.5$; similarity fraction $=0.8$; global alignment $=$ no; auto-detect paired distances $=$ yes; non-specific match handling $=$ map randomly). Subsequently, InDels and structural variants were determined following default parameters ( $p$-value threshold $=0.0001$; maximum number of mismatches $=3$; create breakpoints $=$ no; ignore broken pairs $=$ yes; minimum relative consensus coverage $=0.0$; minimum quality score $=0$; filter variants $=$ no; minimum number of reads $=2$; restrict calling to target regions $=$ not set), and single-nucleotide polymorphisms were determined following default parameters (ploidy $=2$; ignore positions with coverage above $=100,000$; restrict calling to target regions $=$ not set; ignore broken pairs $=$ yes; ignore non-specific matches $=$ reads; minimum coverage $=2$; minimum count $=2$; minimum frequency $(\%)=75.0$; base quality filter $=$ yes; neighborhood radius $=5$; minimum central quality $=20$; minimum neighborhood quality $=15$; read direction filter $=$ yes; direction frequency $(\%)=5.0$; relative read direction filter $=$ yes; significance $(\%)=1.0$; read position filter $=$ no; remove pyroerror variants $=$ no).

Sanger sequencing. Mutated regions were PCR amplified with primers binding upstream and downstream of the mutation using DreamTaq DNA polymerase (Thermo Scientific). PCR products were purified and sent to Eurofins MWG Operon (Ebersberg, Germany) for sequencing.

Strain reconstruction. The reconstructed strains were constructed by using Duplication-Insertion Recombineering ${ }^{57}$ and P22 transduction. A cat-sacB marker (GenBank accession number KM018298) was inserted near each mutation using Lambda-Red recombineering ${ }^{58}$, generating a marker-held tandem duplication. The marker and the linked mutation were then moved using P22 transduction into the recipient strains, and the transductants were re-streaked on salt-free LB agar containing 5\% sucrose. Only bacteria that lose the cat-sacB marker through recombination between the two copies of the duplicated sequence can grow since expression of $s a c B$ is lethal in the presence of sucrose ${ }^{59}$. The transduced mutations were then confirmed by PCR and sequencing. Gene deletions were constructed by replacing the gene with a cat-sacB cassette, and then removing the cassette with a single-stranded DNA oligo in a second round of recombineering. Primer sequences are listed in Supplementary Table 6. Whole-genome sequencing showed that some of the reconstructed strains carried additional, unintended mutations (Supplementary Table 7). Two of them, yebB (S54G) and lon (P12syn), were co-transduced from the isolated mutants, while the rest appeared during the reconstruction process.

Growth rate measurements. Growth rates were measured at $37^{\circ} \mathrm{C}$ in $\mathrm{MH}$ broth using a Bioscreen C Analyzer (Oy Growth Curves Ab Ltd., Helsinki, Finland). Each well was inoculated with a 1000 -fold dilution of an overnight culture and measurements were made in quadruplicates. The cultures were grown for $24 \mathrm{~h}$ with continuous shaking, and $\mathrm{OD}_{600}$ measurements were taken every $4 \mathrm{~min}$. The calculations were based on $\mathrm{OD}_{600}$ values between 0.02 and 0.1 , where growth was observed to be exponential. 
MIC measurements. MIC values were determined by Etest according to the instructions of the manufacturer (bioMérieux, Marcy l'Étoile, France). Etests were performed on $\mathrm{MH}$ agar plates incubated for $16-18 \mathrm{~h}$ at $37^{\circ} \mathrm{C}$. All MIC values presented are the median values of 2-5 Etests from biological replicates.

Spontaneous mutation rates. The mutation rates of the different strains were estimated with fluctuation assays ${ }^{60}$. From frozen stock, the strains were streaked on $\mathrm{MH}$ agar plates and single colonies were used to start overnight cultures in $1 \mathrm{ml} \mathrm{MH}$ medium. These cultures were diluted $10^{3}$ in sterile filtered phosphate-buffered saline and 24 independent cultures of each strain were started with an initial inoculum of approximately $1000 \mathrm{cfu}$ in $1 \mathrm{ml} \mathrm{MH}$ medium. The cultures were incubated in $37^{\circ} \mathrm{C}$ for $20 \mathrm{~h}$ to stationary phase, and rifampinresistant mutants were detected by plating a fraction of each culture on $\mathrm{MH}$ agar medium supplemented with $100 \mathrm{mg} \mathrm{L}^{-1}$ rifampicin. The volumes plated was $50 \mu \mathrm{L}$ for non-mutator strains and $5 \mu \mathrm{L}$ for mutator strains. The population sizes of the cultures were estimated by plating on non-selective $\mathrm{MH}$ agar plates. Plates were incubated for $24 \mathrm{~h}$ in $37^{\circ} \mathrm{C}$ before counting the number of colonies per plate. The estimated mutation rates were calculated with the empirical probability generating function using the web tool bz-rates ${ }^{61}$, with correction for the plating efficiency.

Isolation of total RNA and qRT-PCR. Overnight cultures were diluted 1:100 in $10 \mathrm{ml} \mathrm{MH}$ broth and incubated at $37^{\circ} \mathrm{C}$ to $\mathrm{OD}_{600}=0.4$. To test the effect of excess zinc on aadA expression some cultures were supplemented with $1 \mathrm{mM} \mathrm{ZnCl}_{2} .400$ $\mu \mathrm{l}$ of bacterial culture were mixed with $800 \mu \mathrm{l}$ RNAprotect Bacteria Reagent (Qiagen) and total RNA was extracted using the RNeasy Mini Kit (Qiagen), according to manufacturer's recommendation. Subsequently, the extracted RNA was treated with DNase Turbo DNA-free kit (Amb ion) according to manufacturer's recommendation and subjected to gel electrophoresis to confirm the integrity of the samples. $500 \mathrm{ng}$ of DNase-treated RNA were reverse transcribed using the High Capacity Reverse Transcription kit (Applied Biosystems), according to the manufacturer's recommendation. Relative aadA and iraP transcript levels were determined using PerfeCTa SYBR Green SuperMix (Quanta Biosciences) with the Eco Real-Time PCR System (Illumina). The efficiency of each primer pair was determined in a set of six 10-fold dilutions. Expression levels of aadA and iraP were normalized to the reference genes $h c a T$ and $c y s G$. Primer sequences are listed in Supplementary Table 8 . Measurements were repeated for at least two biological and three technical replicates each.

Streptomycin uptake assay. To determine the concentration of intracellular dihydrostreptomycin an overnight culture of the strain of interest was diluted 1:200 in $\mathrm{MH}$ broth and grown to $\mathrm{OD}_{600}=0.5$. The cultures were supplemented with tritiated dihydrostreptomycin (Larodan, Sweden) to a final activity of $50 \mathrm{nCi} \mathrm{ml}^{-1}$. After $30 \mathrm{~min}, 2 \mathrm{ml}$ were transferred to an Eppendorf tube, washed three times with PBS and finally transferred to $2 \mathrm{ml}$ Optiphase HiSafe 3 (PerkinElmer) scintillation cocktail. Counts per minute were determined using a Tri-Carb 2810 TR liquid scintillation analyzer (PerkinElmer) over a duration of $1 \mathrm{~min}$ per sample.

Model for mutation accumulation and takeover. We consider a deterministic growth model where back mutations and stochastic effects are neglected. Let $N_{n}(t)$ be the number of cells with $n$ selected (by $s$ each) mutations at time $t$, and $M_{n}(t)$ be the corresponding number of mutator cells. They grow with rate $(1+n s) k$. Up to five selected mutations can occur in random order each with the basic rate $u$. Mutators are also assumed to occur with rate $u$. When $n$ selected mutations are present, the next one can occur in $5-n$ ways; hence with total rate $u(5-n)(1+n s)$ $k$. The mutator cells have the basic mutation rate $v$.

$$
\begin{array}{r}
\frac{\mathrm{d} N_{n}}{\mathrm{~d} t}=(1+n s) k N_{n}+u(6-n)[1+(n-1) s] k N_{n-1} \\
\frac{\mathrm{d} M_{n}}{\mathrm{~d} t}=(1+n s) k M_{n}+v(6-n)[1+(n-1) s] k M_{n-1}+u(1+n s) k N_{n} .
\end{array}
$$

With $N_{-1}$ and $M_{-1}=0$ at all times, these equations are valid for $0 \leq n \leq 5$. The initial conditions are:

$$
\begin{aligned}
& N_{0}(0)=N_{\mathrm{e}} \\
& N_{1}(0)=0 \text { or } N_{1}(0)=N_{\mathrm{e}} 5 u / s \\
& N_{n}(0)=0 ; 2 \leq n \leq 5 \\
& M_{n}(0)=0 ; 0 \leq n \leq 5
\end{aligned}
$$

Here, $N_{e}$ is an effective population size. A non-zero initial value for $N_{1}(0)$ is based on the equilibrium value in a population without antibiotics where a mutation will be counter-selected by $-s$. The solution for the non-mutator part of the population

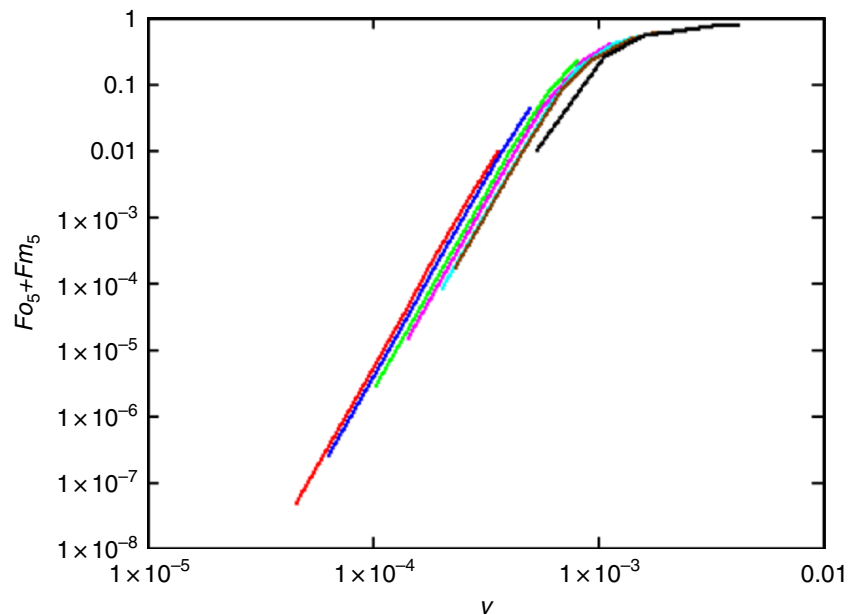

Fig. 7 Penetration of mutants with five selected mutations. Penetration after 900 generations as a function of mutator mutation rate $v$ for values of $u$ between $5 \times 10^{-7}$ (black curve on the right) and $4 \times 10^{-6}$ (red curve on the left)

is

$$
\begin{gathered}
N_{0}(t)=N_{\mathrm{e}} \mathrm{e}^{k t} \\
N_{n}(t)=N_{\mathrm{e}} \mathrm{e}^{(1+n s) k t}\left(\begin{array}{c}
5 \\
n
\end{array}\right)\left(\frac{u}{s}\right)^{n} \sum_{j=0}^{n-1}(1+j s)\left[\left(1-\mathrm{e}^{-s k t}\right)^{n}+A n\left(1-\mathrm{e}^{-s k t}\right)^{n-1}\right] \\
1 \leq n \leq 5
\end{gathered}
$$

The parameter $A=0$ is for the case with $N_{1}(0)=0$ and $A=1$ corresponds to the case where $N_{1}(0)=N_{e} 5 u / s$. The mutators are most easily handled by numerical integration. Let

$$
M_{n}(t)=\mathrm{e}^{(1+n s) k t} g_{n}(t)
$$

Then

$$
g_{0}(t)=N_{\mathrm{e}} u t
$$

$$
\begin{gathered}
\frac{\mathrm{d} g_{n}}{\mathrm{~d} t}=v(6-n)[1+(n-1) s] k \mathrm{e}^{-s t} g_{n-1}(t)+u(1+n s) k \mathrm{e}^{-(1+n s) k t} N_{n}(t) ; \\
1 \leq n \leq 5
\end{gathered}
$$

The fractions of the different variants at any time can be calculated from

$$
\mathrm{Fo}_{n}=N_{n} / \sum_{n}\left(N_{n}+M_{n}\right) \text { and } \mathrm{Fm}_{n}=M_{n} / \sum_{n}\left(N_{n}+M_{n}\right)
$$

In the calculations, it has been assumed that all selected mutations contribute independently and that each has the same selection coefficient $s=0.006$, such that five of them give a $3 \%$ growth advantage in the presence of the antibiotic. Furthermore, a mutator mutation is assumed to occur with the same rate, $u$, as each of the selected ones. It is straightforward to expand the calculations to include a non-zero initial presence of mutators. However, with an expected fraction on the order of $10^{-6}$, this is found to have a very small effect on the results and is not considered further. The expected initial fraction of single mutants, $5 u / s$, may be on the order of $10^{-3}$, which can contribute significantly. Variants with two or more mutations are expected to be present in insignificant fractions initially. Mutators are expected to accumulate deleterious mutations over time contributing to some growth disadvantage for them. Here it is assumed that this effect is small on the time scale considered. Finally, the growth rate $k=\ln (2)$ if time, $t$, is considered in number of generations (doublings). Figure 5 summarizes the time development of the different mutant types.

Without mutators, $1 \%$ penetration after 900 generations can be achieved only with a very high mutation rate, $u=9.5 \times 10^{-4}$, or slightly lower, $u=7.4 \times 10^{-4}$, if there is a fraction $5 \mathrm{u} / \mathrm{s}$ of single mutants present initially. When mutators contribute, this initial condition has little effect and has not been included in the 
calculations of the results shown in Fig. 7 and Table 3. We have varied the mutation rate $u$ ca. 10 -fold between $5 \times 10^{-7}$ and $4 \times 10^{-6}$ and the ratio $v / u$ also 10 -fold between ca. $10^{2}$ and $10^{3}$. It is noteworthy that throughout these ranges, $1 \%$ penetration is reached with a mutator rate $v$ of ca. $(3.5-5) \times 10^{-4}$.

\section{Calculating epistasis using the weighted Walsh-Hadamard transform. We} studied five different genetic loci with either the wild-type or a mutant sequence, making a total of $2^{5}=32$ genotypes, so the collection of all 32 reconstructed mutants with resistance phenotypes (Fig. 1 and Table 2) represents a combinatorially complete dataset for the five mutations. The fitness landscape can be seen as a high-dimensional space where different orders of epistatic (non-additive) interactions can be calculated through spectral analysis using transform theory 62,63 , similarly to how a Fourier transform can be used to decompose a signal in the time domain into different components in the frequency domain. The higher-order epistatic effects of the different resistance mutations were calculated through Walsh-Hadamard transform decomposition ${ }^{64}$ of the fitness landscape of streptomycin resistance. Since this is a linear epistasis model while antibiotic resistance increases in a multiplicative way, the phenotypic resistance values were linearized as $\log _{2}$ (MIC).

The epistatic effects were calculated in two different ways; as the global epistatic effect of each mutation (background-averaged epistasis, corresponding to a Fourier expansion), and as the epistatic effect of each mutation with the wild-type sequence as reference (corresponding to a Taylor expansion) ${ }^{64}$. Interactions between the different mutations that are purely additive will have epistatic coefficients equal to zero, while epistatic interactions will be non-zero. The epistasis coefficients of each order are calculated so that higher-order epistasis coefficients only represent the variation that cannot be described as the sum of the lower-order effects. The mathematical theory behind the model has been thoroughly discussed by Poelwijk et al. ${ }^{64}$, so we will only give a summary of the model here.

If we define $\overline{\boldsymbol{\omega}}$ as the vector of $2^{n}$ epistasis coefficients of all orders, $\bar{y}$ as the vector corresponding to phenotypes $y\left(\log _{2}(\mathrm{MIC})\right)$ of all the individual variants listed in binary order (Supplementary Table 2), and the $2^{n} \times 2^{n}$ matrix $\boldsymbol{\Omega}_{\text {epi }}$ as the epistasis transform, we can write

$$
\overline{\boldsymbol{\omega}}=\Omega_{\mathrm{epi}} \bar{y} .
$$

We first calculated the global, background-averaged epistasis $\varepsilon$; the epistatic effects of each mutation as an average over all possible sequence variants. In this case, we can define

$$
\boldsymbol{\Omega}_{\text {epi_global }}=\boldsymbol{V H},
$$

where $V$ is a weighting matrix to normalize for the different numbers of terms for epistasis of different orders, recursively defined as

$$
\boldsymbol{V}_{n+1}=\left(\begin{array}{cc}
\frac{1}{2} \boldsymbol{V}_{n} & 0 \\
0 & \boldsymbol{V}_{n}
\end{array}\right) \text { with } \boldsymbol{V}_{0}=1
$$

and the matrix $\boldsymbol{H}$ corresponds to the Walsh-Hadamard transform ${ }^{63,64}$ generated by the recursive definition

$$
\boldsymbol{H}_{n+1}=\left(\begin{array}{cc}
\boldsymbol{H}_{n} & \boldsymbol{H}_{n} \\
\boldsymbol{H}_{n} & -\boldsymbol{H}_{n}
\end{array}\right) \text { with } \boldsymbol{H}_{0}=1
$$

The vector of background-averaged epistatic terms $\bar{\varepsilon}$ can then be calculated by

$$
\bar{\varepsilon}=V H \bar{y} .
$$

To calculate the vector of epistatic terms $\bar{\lambda}$ relative to the wild-type sequence, we instead use

$$
\Omega_{\text {epi_wt }}=V X^{T} H
$$

where $\boldsymbol{X}^{T}$ only includes the terms associated with the wild type, and the matrix $\boldsymbol{X}$ can be defined as

$$
\boldsymbol{X}_{n+1}=\left(\begin{array}{cc}
\boldsymbol{X}_{n} & 0 \\
\boldsymbol{X}_{n} & \boldsymbol{X}_{n}
\end{array}\right) \text { with } \boldsymbol{X}_{0}=1
$$

which gives us

$$
\bar{\lambda}=V \boldsymbol{X}^{T} \boldsymbol{H} \bar{y} .
$$

The calculations were performed using Python 3.6 with the NumPy library.

Code availability. Python scripts are available from the corresponding author upon request.
Data availability. All WGS data that support the findings of this study have been deposited in the NCBI SRA and are accessible through the accession no. SRP133288. All other relevant data are available from the corresponding author upon request.

Received: 21 November 2017 Accepted: 29 March 2018

Published online: 23 April 2018

\section{References}

1. Andersson, D. I. \& Hughes, D. Microbiological effects of sublethal levels of antibiotics. Nat. Rev. Microbiol. 12, 465-478 (2014).

2. Thiele-Bruhn, S. Pharmaceutical antibiotic compounds in soils-a review. J. Plant Nutr. Soil Sci. 166, 145-167 (2003).

3. Chander, Y., Kumar, K., Goyal, S. M. \& Gupta, S. C. Antibacterial activity of soil-bound antibiotics. J. Environ. Qual. 34, 1952-1957 (2005).

4. Martínez, J. L. Antibiotics and antibiotic resistance genes in natural environments. Science 321, 365-367 (2008).

5. Kümmerer, K. Antibiotics in the aquatic environment-a review. Chemosphere 75, 417-434 (2009).

6. Khan, G. A., Berglund, B., Khan, K. M., Lindgren, P.-E. \& Fick, J. Occurrence and abundance of antibiotics and resistance genes in rivers, canal and near drug formulation facilities-a study in Pakistan. PLoS ONE 8, e62712 (2013).

7. Lindberg, R. H. et al. Environmental risk assessment of antibiotics in the Swedish environment with emphasis on sewage treatment plants. Water Res. 41, 613-619 (2007)

8. Gullberg, E. et al. Selection of resistant bacteria at very low antibiotic concentrations. PLoS Pathog. 7, e1002158 (2011).

9. Liu, A. et al. Selective advantage of resistant strains at trace levels of antibiotics: a simple and ultrasensitive color test for detection of antibiotics and genotoxic agents. Antimicrob. Agents Chemother. 55, 1204-1210 (2011).

10. Gullberg, E., Albrecht, L. M., Karlsson, C., Sandegren, L. \& Andersson, D. I. Selection of a multidrug resistance plasmid by sublethal levels of antibiotics and heavy metals. mBio 5, e01918 (2014).

11. Bottery, M. J., Wood, A. J. \& Brockhurst, M. A. Selective conditions for a multidrug resistance plasmid depend on the sociality of antibiotic resistance. Antimicrob. Agents Chemother. 60, 2524-2527 (2016).

12. Lundström, S. V. et al. Minimal selective concentrations of tetracycline in complex aquatic bacterial biofilms. Sci. Total Environ. 553, 587-595 (2016).

13. Khan, S., Beattie, T. K. \& Knapp, C. W. The use of minimum selectable concentrations (MSCs) for determining the selection of antimicrobial resistant bacteria. Ecotoxicology 26, 283-292 (2017)

14. Jørgensen, K. M. et al. Sublethal ciprofloxacin treatment leads to rapid development of high-level ciprofloxacin resistance during long-term experimental evolution of Pseudomonas aeruginosa. Antimicrob. Agents Chemother. 57, 4215-4221 (2013).

15. Oz, T. et al. Strength of selection pressure is an important parameter contributing to the complexity of antibiotic resistance evolution. Mol. Biol. Evol. 31, 2387-2401 (2014).

16. Chow, L., Waldron, L. \& Gillings, M. R. Potential impacts of aquatic pollutants: sub-clinical antibiotic concentrations induce genome changes and promote antibiotic resistance. Front. Microbiol. 6, 803 (2015).

17. Wassermann, T. et al. The phenotypic evolution of Pseudomonas aeruginosa populations changes in the presence of subinhibitory concentrations of ciprofloxacin. Microbiology 162, 865-875 (2016).

18. Westhoff, S. et al. The evolution of no-cost resistance at sub-MIC concentrations of streptomycin in Streptomyces coelicolor. ISME J. 11, 1168-1178 (2017)

19. Zaborskyte, G., Andersen, J. B., Kragh, K. N. \& Ciofu, O. Real-time monitoring of $\mathrm{nfxB}$ mutant occurrence and dynamics in Pseudomonas aeruginosa biofilm exposed to subinhibitory concentrations of ciprofloxacin. Antimicrob. Agents Chemother. 61, e02292 (2017).

20. Ozaki, M., Mizushima, S. \& Nomura, M. Identification and functional characterization of the protein controlled by the streptomycin-resistant locus in E. coli. Nature 222, 333-339 (1969).

21. Funatsu, G. \& Wittmann, H. G. Ribosomal proteins. 33. Location of aminoacid replacements in protein S12 isolated from Escherichia coli mutants resistant to streptomycin. J. Mol. Biol. 68, 547-550 (1972).

22. Gill, A. E. \& Amyes, S. G. B. The contribution of a novel ribosomal S12 mutation to aminoglycoside resistance of Escherichia coli mutants. J. Chemother. 16, 347-349 (2004)

23. Spagnolo, F., Rinaldi, C., Sajorda, D. R. \& Dykhuizen, D. E. Evolution of resistance to continuously increasing streptomycin concentrations in populations of Escherichia coli. Antimicrob. Agents Chemother. 60, 1336-1342 (2015). 
24. Pränting, M. \& Andersson, D. I. Mechanisms and physiological effects of protamine resistance in Salmonella enterica serovar Typhimurium LT2. J. Antimicrob. Chemother. 65, 876-887 (2010).

25. Koskiniemi, S., Pränting, M., Gullberg, E., Näsvall, J. \& Andersson, D. I. Activation of cryptic aminoglycoside resistance in Salmonella enterica. Mol. Microbiol. 80, 1464-1478 (2011).

26. Proctor, R. A. et al. Small colony variants: a pathogenic form of bacteria that facilitates persistent and recurrent infections. Nat. Rev. Microbiol. 4, 295-305 (2006).

27. Knöppel, A., Näsvall, J. \& Andersson, D. I. Evolution of antibiotic resistance without antibiotic exposure. Antimicrob. Agents Chemother. 61, 01495 (2017).

28. Horst, J. P., Wu, T. H. \& Marinus, M. G. Escherichia coli mutator genes. Trends Microbiol. 7, 29-36 (1999).

29. Okamoto, S. et al. Loss of a conserved 7-methylguanosine modification in $16 \mathrm{~S}$ rRNA confers low-level streptomycin resistance in bacteria. Mol. Microbiol. 63, 1096-1106 (2007).

30. Lázár, V. et al. Bacterial evolution of antibiotic hypersensitivity. Mol. Syst. Biol. 9, 700 (2013).

31. Lenski, R. E. et al. Evolution of competitive fitness in experimental populations of $E$. coli: what makes one genotype a better competitor than another? Antonie van Leeuwenhoek 73, 35-47 (1998).

32. Dardonville, B. \& Raibaud, O. Characterization of malT mutants that constitutively activate the maltose regulon of Escherichia coli. J. Bacteriol. 172, 1846-1852 (1990).

33. Bryan, L. E. \& Van den Elzen, H. M. Streptomycin accumulation in susceptible and resistant strains of Escherichia coli and Pseudomonas aeruginosa. Antimicrob. Agents Chemother. 9, 928-938 (1976).

34. Campbell, B. D. \& Kadner, R. J. Relation of aerobiosis and ionic strength to the uptake of dihydrostreptomycin in Escherichia coli. Biochim. Biophys. Acta 593, 1-10 (1980).

35. Bryan, L. E. \& Kwan, S. Roles of ribosomal binding, membrane potential, and electron transport in bacterial uptake of streptomycin and gentamicin. Antimicrob. Agents Chemother. 23, 835-845 (1983).

36. Taber, H. W., Mueller, J. P., Miller, P. F. \& Arrow, A. S. Bacterial uptake of aminoglycoside antibiotics. Microbiol. Rev. 51, 439-457 (1987).

37. Rhoads, D. B. \& Epstein, W. Energy coupling to net $\mathrm{K}+$ transport in Escherichia coli K-12. J. Biol. Chem. 252, 1394-1401 (1977).

38. Pränting, M., Negrea, A., Rhen, M. \& Andersson, D. I. Mechanism and fitness costs of PR-39 resistance in Salmonella enterica serovar Typhimurium LT2. Antimicrob. Agents Chemother. 52, 2734-2741 (2008).

39. Koskiniemi, S., Hughes, D. \& Andersson, D. I. Effect of translesion DNA polymerases, endonucleases and RpoS on mutation rates in Salmonella typhimurium. Genetics 185, 783-795 (2010).

40. Taddei, F. et al. Role of mutator alleles in adaptive evolution. Nature 387, 700-702 (1997).

41. Chao, L. \& Cox, E. C. Competition between high and low mutating strains of Escherichia coli. Evolution 37, 125-134. (1983).

42. Mao, E. F., Lane, L., Lee, J. \& Miller, J. H. Proliferation of mutators in A cell population. J. Bacteriol. 179, 417-422 (1997).

43. Tenaillon, O., Taddei, F., Radmian, M. \& Matic, I. Second-order selection in bacterial evolution: selection acting on mutation and recombination rates in the course of adaptation. Res. Microbiol. 152, 11-16 (2001).

44. Block, R. \& Haseltine, A. W. Purification and properties of stringent factor. J. Biol. Chem. 250, 1212-1217 (1975)

45. Murray, K. D. \& Bremer, H. Control of spoT-dependent ppGpp synthesis and degradation in Escherichia coli. J. Mol. Biol. 259, 41-57 (1996).

46. Patzer, S. I. \& Hantke, K. The ZnuABC high-affinity zinc uptake system and its regulator Zur in Escherichia coli. Mol. Microbiol. 28, 1199-1210 (1998).

47. Patzer, S. I. \& Hantke, K. The zinc-responsive regulator Zur and its control of the znu gene cluster encoding the ZnuABC zinc uptake system in Escherichia coli. J. Biol. Chem. 275, 24321-24332 (2000).

48. Coleman, J. E. Zinc enzymes. Curr. Opin. Chem. Biol. 2, 222-234 (1998).

49. Bougdour, A. \& Gottesman, S. ppGpp regulation of RpoS degradation via anti-adaptor protein IraP. Proc. Natl Acad. Sci. USA 104, 12896-12901 (2007).

50. Paul, B. J., Berkmen, M. B. \& Gourse, R. L. DksA potentiates direct activation of amino acid promoters by ppGpp. Proc. Natl Acad. Sci. USA 102, 7823-7828 (2005).

51. Lemke, J. J., Durfee, T. \& Gourse, R. L. DksA and ppGpp directly regulate transcription of the Escherichia coli flagellar cascade. Mol. Microbiol. 74, 1368-1379 (2009).

52. Furman, R. et al. $\mathrm{pH}$ dependence of the stress regulator DksA. PLoS ONE 10, e0120746 (2015).
53. Perederina, A. et al. Regulation through the secondary channel-structural framework for ppGpp-DksA synergism during transcription. Cell 118 297-309 (2004)

54. Perron, K., Comte, R. \& van Delden, C. DksA represses ribosomal gene transcription in Pseudomonas aeruginosa by interacting with RNA polymerase on ribosomal promoters. Mol. Microbiol. 56, 1087-1102 (2005).

55. Blaby-Haas, C. E., Furman, R., Rodionov, D. A., Artsimovitch, I. \& de CrécyLagard, V. Role of a Zn-independent DksA in Zn homeostasis and stringent response. Mol. Microbiol. 79, 700-715 (2011).

56. Melnyk, A. H., McCloskey, N., Hinz, A. J., Dettman, J. \& Kassen, R. Evolution of cost-free resistance under fluctuating drug selection in Pseudomonas aeruginosa. mSphere 2, e00158 (2017).

57. Näsvall, J., Knöppel, A. \& Andersson, D. I. Duplication-insertion recombineering: a fast and scar-free method for efficient transfer of multiple mutations in bacteria. Nucleic Acids Res. 45, e33 (2016).

58. Datsenko, K. A. \& Wanner, B. L. One-step inactivation of chromosomal genes in Escherichia coli K-12 using PCR products. Proc. Natl Acad. Sci. USA 97, 6640-6645 (2000).

59. Ried, J. L. \& Collmer, A. An nptI-sacB-sacR cartridge for constructing directed, unmarked mutations in gram-negative bacteria by marker exchangeeviction mutagenesis. Gene 57, 239-246 (1987).

60. Rosche, W. A. \& Foster, P. L. Determining mutation rates in bacterial populations. Methods 20, 4-17 (2000).

61. Gillet-Markowska, A., Louvel, G. \& Fischer, G. bz-rates: a web tool to estimate mutation rates from fluctuation analysis. G3 5, 2323-2327 (2015).

62. Neidhart, J., Szendro, I. G. \& Krug, J. Exact results for amplitude spectra of fitness landscapes. J. Theor. Biol. 332, 218-227 (2013).

63. Weinreich, D. M., Lan, Y., Wylie, C. S. \& Heckendorn, R. B. Should evolutionary geneticists worry about higher-order epistasis? Curr. Opin. Genet. Dev. 23, 700-707 (2013).

64. Poelwijk, F. J., Krishna, V. \& Ranganathan, R. The context-dependence of mutations: a linkage of formalisms. PLoS Comp. Biol. 12, e1004771 (2016).

\section{Acknowledgements}

This work was supported by grants from the Swedish Research Council and Formas to D.I.A. We are grateful to Po-Cheng Tang for his advice on the bioinformatics work in this manuscript.

\section{Author contributions}

D.I.A., E.W.Y., and M.K. designed the experiments; E.W.Y., M.K., K.H., and S.K. performed the experiments. E.W.Y., M.K., O.G.B., D.I.A., K.H., and S.K. analyzed the data E.W.Y., M.K., and D.I.A. wrote the manuscript with contributions from all authors.

\section{Additional information}

Supplementary Information accompanies this paper at https://doi.org/10.1038/s41467 018-04059-1.

Competing interests: The authors declare no competing interests.

Reprints and permission information is available online at http://npg.nature.com/ reprintsandpermissions/

Publisher's note: Springer Nature remains neutral with regard to jurisdictional claims in published maps and institutional affiliations.

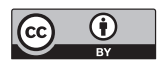

Open Access This article is licensed under a Creative Commons Attribution 4.0 International License, which permits use, sharing, adaptation, distribution and reproduction in any medium or format, as long as you give appropriate credit to the original author(s) and the source, provide a link to the Creative Commons license, and indicate if changes were made. The images or other third party material in this article are included in the article's Creative Commons license, unles indicated otherwise in a credit line to the material. If material is not included in the article's Creative Commons license and your intended use is not permitted by statutory regulation or exceeds the permitted use, you will need to obtain permission directly from the copyright holder. To view a copy of this license, visit http://creativecommons.org/ licenses/by/4.0/.

(C) The Author(s) 2018 